\title{
Analysis of Low Energy Static Posture for Hexapod Bionic Robot
}

\author{
Lei Zhang, Fang Yang*, Dedong Li, Juncheng Guo and Cechong Liu \\ College of Engineering, Ocean University of China, China \\ *Corresponding author
}

\begin{abstract}
According to the workload requirements of multilegged robots, the standing posture of hexapod bionic robot based on static analysis is proposed in this paper, which aims to reduce the energy consumption. We chose the picnic insects with low speed as the bionic prototype and build the robot model, Through the statics analysis we get the low energy static posture when the output torque of the robot is smallest. On this basis, a multiobjective optimization method based on energy consumption and space flexibility is proposed. Finally, we design the simulation and prototype test on self-developed hexapod robot, the results show that the standing posture proposed in this paper satisfies the requirements of energy consumption and space flexibility.
\end{abstract}

Keywords-low energy; hexapod bionic robot; static analysis; joint torque; optimization simulation

\section{INTRODUCTION}

Multi-legged robot has rich form of motion, high bearing capacity and strong stability[1]. Based on the observation that the body of ants, spiders and other insects system consume low energy when they in the quiescent state[2], this paper presents a low-power posture of hexapod robot, which directly affects the stability and reliability of the robot[3].

Early scholars using the Newton Euler method analyzed the static heat loss of the robot and put forward the optimization allocation method for six legged robot output torque[4], but this method is limited to evaluate the energy consumption of the system by computer simulation, which lack of further analysis on the effective power of the actual robot when standing[5]. Then SHIBENDU S R [6]established the dynamic equations of the Lagrange Euler system and using the least squares method got the robot joint torque and optimization trajectory, but the calculation is too complicated to achieve.

In this paper, the single leg of the hexapod robot has three degrees of freedom is taken as the object of study[7]. Based on the static model, a method for analyzing the low power static posture of multi-legged robot and establish the multi-objective function of energy consumption and motion flexibility of robot to optimize the posture of the robot[8]. Then the simulation results of the low energy static posture and the prototype experiment are given[9]. This provides a theoretical basis for the motion control of the robot and provides reference for the design of the hexapod robot mechanism.

\section{STATICS ANALYSIS OF HEXAPOD ROBOT}

\section{A. Modeling of Hexapod Robot}

At present, the mechanical design of the hexapod robot is based on the theory of bionics. Through observation and summary, the insects has six legs has better adaptability and workload, and stick insect is one of the most representative biology. Summary the law of motion and structure characteristics of these insects, this paper establishes a model of insect like six legged robot. The model is a kind of high redundancy structure system with series parallel connection and six legs evenly distributed in both sides of the body, each leg is composed of three segments (coxa, femur and tibia) and three revolute joints (root joint, hip joint and knee joint) from body to the foot end. In order to description detailedly, this paper numbers the six legs $1,3,5,2,4,6$, corresponding to $L_{1}, L_{2}, L_{3}, R_{1}, R_{2}, R_{3}$. The structure of the model is shown in figure 1.

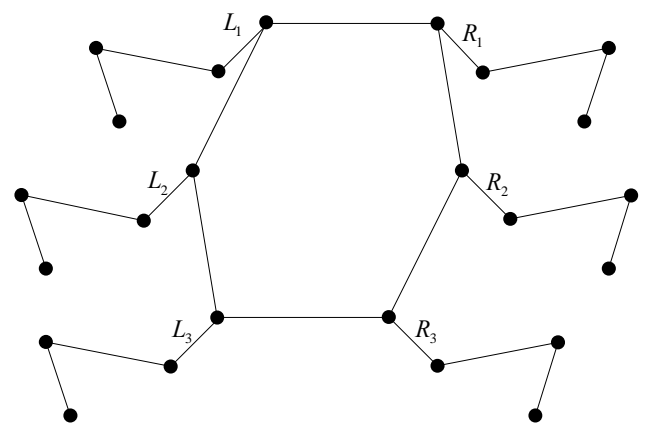

FIGURE I. MODEL OF THE HEXAPOD BIONIC ROBOT.

\section{B. Static Analysis of Single Leg}

According to the structure of a bionic model of hexapod robot in Figure 1, six legs of the robot are symmetrical. We select leg 3 and 4 as the research objects, the establishment of the static model and coordinate system are shown in figure3. The origin of the static coordinate system is the center of the robot, the $x$ axis is perpendicular to the ground, and the $z$ axis points to the right side of the body. The main stress concentrated in the $x-z$ plane when robot is static. Therefore, in the static analysis, the revolute pair of the leg is regarded as a fixed joint in the $x-y$ plane and the driving force of robot joint is looked as the corresponding joint driving torque. 
Among them A, B are centers of mass of femur and tibia respectively, $\theta_{2}, \theta_{3}$ denote the angles of hip joint and knee joint. $M_{2}, M_{3}$ denote the torque of the hip joint and knee joint, $m_{2}, m_{3}$ denote quality of femur and tibia, the length of femur and tibia are described by $l_{1}, l_{2}$, and $P$ is the supporting power, $p^{\prime}$ is the foot endpoint.

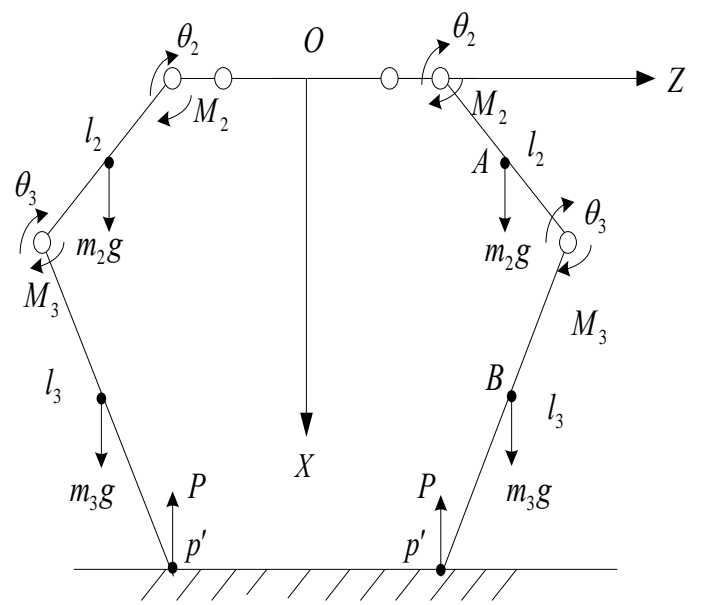

FIGURE II. FORCE ANALYSIS OF TWO LEGS IN $x-z$ PLANE

We select $\theta_{2}, \theta_{3}$ as the generalized coordinates, then the coordinates of the $\mathrm{X}$ axes of each particle can be expressed as:

$$
\left\{\begin{array}{l}
x_{A}=\frac{1}{2} l_{2} \sin \theta_{2} \\
x_{B}=l_{2} \sin \theta_{2}+\frac{1}{2} l_{3} \sin \left(\theta_{2}+\theta_{3}\right) \\
x_{P}=l_{2} \sin \theta_{2}+l_{3} \sin \left(\theta_{2}+\theta_{3}\right)
\end{array}\right.
$$
by:

Then balance equation of virtual displacement can be given

$$
\begin{aligned}
& M_{2}+\frac{\partial x_{A}}{\partial \theta_{2}} m_{2} g+\frac{\partial x_{B}}{\partial \theta_{2}} m_{3} g-\frac{\partial x_{P}}{\partial \theta_{2}} P=0 \\
& M_{3}+\frac{\partial x_{B}}{\partial \theta_{3}} m_{3} g-\frac{\partial x_{P}}{\partial \theta_{3}} P=0
\end{aligned}
$$

Therefore, torques of the hip joint and knee joint can be expressed as:

$$
\begin{aligned}
M_{2}= & P\left[l_{2} \cos \theta_{2}+l_{3} \cos \left(\theta_{2}+\theta_{3}\right)\right]-\frac{1}{2} m_{2} l_{2} g \cos \theta_{2} \\
& -m_{3} g\left[l_{2} \cos \theta_{2}+\frac{1}{2} l_{3} \cos \left(\theta_{2}+\theta_{3}\right)\right]
\end{aligned}
$$

$$
M_{3}=-\frac{1}{2} m_{3} l_{3} g \cos \left(\theta_{2}+\theta_{3}\right)+P l_{3} \cos \left(\theta_{2}+\theta_{3}\right)
$$

When the robot is standing still, all legs are in contact with the ground, we assuming that the robot is static and the ground supporting force of each foot is equal. From the above description, it can be concluded that the single leg energy consumption can be expressed as the sum of the output torque of the hip joint and knee joint when the robot is standing still. The low energy consumption of the system can be obtained when the output torque is the smallest.

\section{Optimization of LOW Energy Static Posture}

The setting of the posture of the six legged robot not only to consider the energy consumption, but also the maximum flexibility which is the measure standard whether there is enough space for the movement when the robot in the state from the static to the movement. Therefore, the optimization of the posture becomes to solve the two problem of the maximum distance and energy consumption. The calculation of energy consumption and maximum step distance of the robot can be described as:

- $\quad$ Energy consumption of the single leg of the robot:

$$
M=\left|M_{2}\right|+\left|M_{3}\right|
$$

- $\quad$ Maximum step distance:

First of all, the model of leg 6 and the body in the xoy plane can be given:

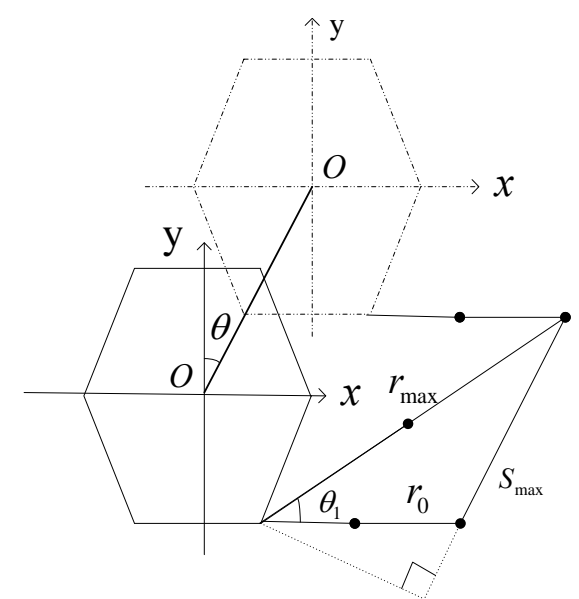

FIGURE III. THE ROBOT MODEL OF LEG 6 AND THE BODY

The solid and dashed lines are the initial position and the end position in a gait cycle of the robot. $\theta$ is course angle of the robot. $r_{\max }$ and $r_{0}$ are projection length on the xoy plane of the robot when the legs are at maximum and initial state. Based on Figure 3, we can get maximum step distance from the geometrical relations: 


$$
\begin{aligned}
S_{\max } & =\sqrt{\begin{array}{l}
\left(l_{1}+\sqrt{\left(l_{2}+l_{3}\right)^{2}-H^{2}}\right)^{2}- \\
\left(l_{1}+l_{2} \cos \theta_{2}+l_{3} \cos \left(\left|\theta_{3}\right|-\theta_{2}\right)\right)^{2} \cos ^{2} \theta
\end{array}} \\
& -\left(l_{1}+l_{2} \cos \theta_{2}+l_{3} \cos \left(\left|\theta_{3}\right|-\theta_{2}\right)\right) \sin \theta
\end{aligned}
$$

From viewpoint of easy realization, weighted sum algorithm is used to solve multi-objective optimization problems in this paper. The mathematical description of the problem is shown in (7). $M$ is minimum power target and the expression of power consumption is shown in (5) when the robot is standing still. $S_{\max }$ is the maximum step target and it is shown in (6).

In order to calculate the expression conveniently, this paper introduces the concept of closeness degree. The proximity function is set to $f(x) . k_{1}, k_{2}$ are weighting coefficients, which satisfy the relation of $k_{1}+k_{2}=1, k_{1}, k_{2} \in[0,1]$. Their values are artificially set according to requirements. The range of $f(x)$ is $[0,1] . f(x)$ has no unit, and it means the extent that the posture is close to the optimal posture.

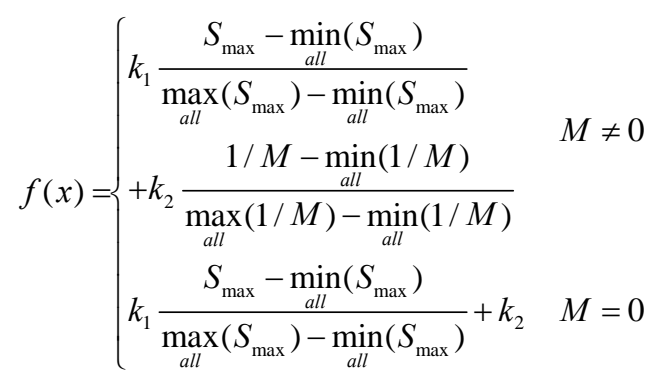

\section{SimUlation AND PROTOTYPE EXPERIMENT}

In this paper, the six legged bionic robot designed independently is as the simulation and experiment object. The robot single leg is seen as three bar linkage and number 1,2,3 correspondingly represent coxal, femur, tibia of the robot. Its mechanism parameters are shown in Table1.

TABLE I. MECHANISM PARAMETERS OF HEXAPOD ROBOT

\begin{tabular}{cccc}
\hline $\begin{array}{c}\text { Rod } \\
\text { Number }\end{array}$ & $\begin{array}{c}\text { Length } \\
l_{i}(\mathbf{m m})\end{array}$ & $\begin{array}{c}\text { Weight } \\
m_{i} \mathbf{( g )}\end{array}$ & $\begin{array}{c}\text { Joint Angle } \\
\left.\boldsymbol{\theta}_{i} \mathbf{(}^{\circ}\right)\end{array}$ \\
\hline 1 & 75 & 42 & $-45 \sim 45$ \\
2 & 105 & 44 & $-45 \sim 90$ \\
3 & 145 & 40 & $-120 \sim 0$ \\
\hline
\end{tabular}

\section{A. Simulation Experiment}

According to the actual mechanism, the robot center of gravity $H$ is set to $110 \mathrm{~mm}$. According to the relationship of the robot center of gravity and the angle joints, the actual range of $\theta_{2}$ is $[-0.4554,0.3346]$, and $\theta_{3}$ is [-1.8208, 0]. $\theta_{2}$ and $\theta_{3}$ are one to one. The corresponding relation can be given:

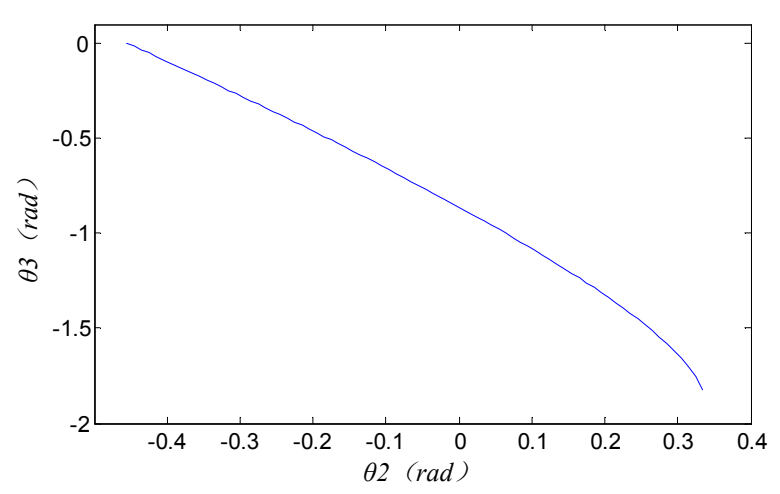

FIGURE IV. RELATIONSHIP BETWEEN $\theta_{2}$ AND $\theta_{3}$

From (6) and (7) we can know that the energy consumption and the maximum step distance of the robot in stationary state are only related to $\theta_{2}, \theta_{3}$ and not $\theta_{1}$. According to the characteristics of the robot, $\theta_{1}$ is set to 0 when the robot is in a low power consumption standing posture. We can know from above that $\theta_{2}$ and $\theta_{3}$ are one to one, so $f(x)$ can be regarded as a function only relate to the dependent variable $\theta_{2}$ (or $\theta_{3}$ ) . We can get their relationship as shown in Figure5. The circle calibration point represents that when $f(x)$ is 0.9 . It is described as the most advantageous point and the corresponding joint angles are $\theta_{2}=0.2146, \theta_{3}=-1.3648$.

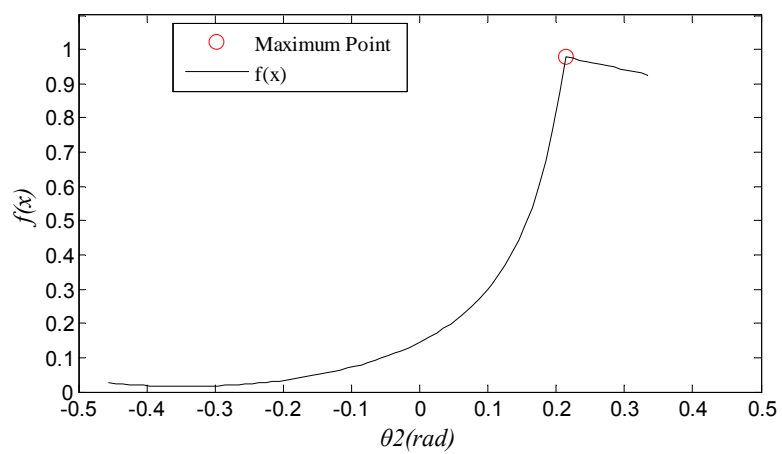

FIGURE V. RELATIONSHIP CURVE OF $f(x)$ AND JIONT ANGLE

In order to verify that the posture can meet the requirements of low energy consumption and flexibility, in this paper, the torque and maximum step are compared between low energy posture and other postures. The change of total joint torque under different posture is shown in Figure 6. The dotted line is the angle of the joint under the posture obtained in this paper. It is shown that the power consumption of the robot is the lowest.

Similarly, the maximum step change of the robot under different postures is shown in Figure 7. As can be seen from the figure, although the maximum step is not the largest in the whole angle range, but it is also greater than most step length under other postures. The maximum step length is $183.373 \mathrm{~mm}$, as shown in the red dotted line. 

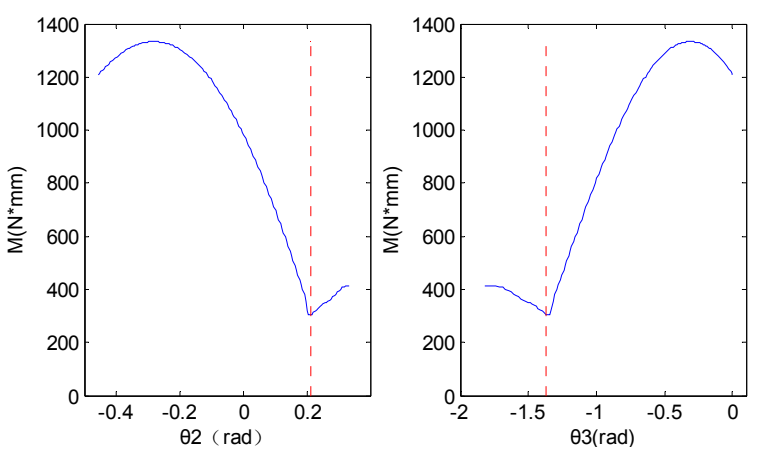

FIGURE VI. SIMULATION CURVE BETWEEN JOINT TORQUE AND TURNING ANGLE
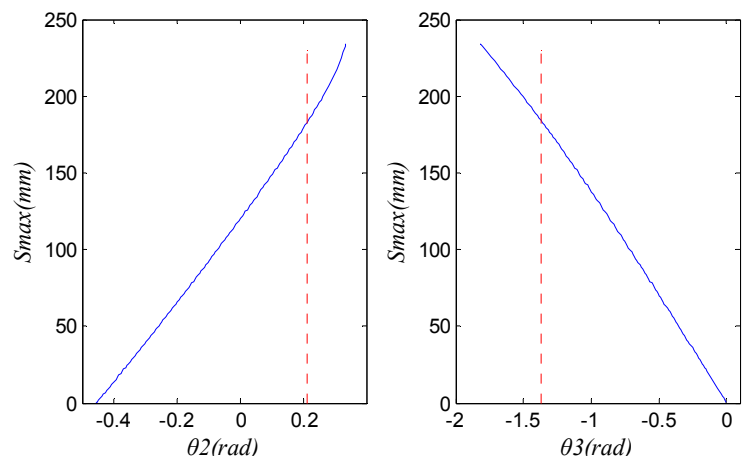

FIGURE VII. SIMULATION CURVE BETWEEN STEP LENGTH AND TURNING ANGLE

\section{B. Prototype Experiment}

In order to verify the correctness of the low power standing posture, this paper selects several typical standing postures to do the physical energy consumption test. The experimental center of gravity height is set to $110 \mathrm{~mm}$, and we use lithium battery of $4200 \mathrm{mAh}$. The experimental results are shown in Table 2.

\section{TABLE II. PROTOTYPE EXPERIMENTAL RESULTS OF ENERGY CONSUMPTION}

\begin{tabular}{ccccc}
\hline $\begin{array}{c}\text { Posture } \\
\text { Number }\end{array}$ & $\begin{array}{c}\text { Joint Angle } \\
\left(\theta_{1}, \theta_{2}, \theta_{3}\right)\end{array}$ & $\begin{array}{c}\text { Step } \\
\text { Length } \\
(\mathbf{m m})\end{array}$ & $\begin{array}{c}\text { Standin } \\
\text { g Time } \\
\mathbf{( h )}\end{array}$ & $\begin{array}{c}\text { Total } \\
\text { Current } \\
(\mathbf{m A})\end{array}$ \\
\hline Posture 1 & $(0,0.3346,-1.8208)$ & 234.4 & 1.04 & 3940 \\
Posture 2 & $(0,0.2746,-1.5442)$ & 204.2 & 1.15 & 3600 \\
Posture 3 & $(0,0.2046,-1.3377)$ & 180.1 & 1.19 & 3510 \\
Posture 4 & $(0,0.1546,-1.2101)$ & 164.6 & 1.02 & 4100 \\
Posture 5 & $(0,0.0946,-1.0684)$ & 146.8 & 0.96 & 4300 \\
Posture 6 & $(0,-0.2854,-0.3027)$ & 43.0 & 0.92 & 4560 \\
Posture 7 & $(0,0.2146,-1.3648)$ & 183.3 & 1.2 & 3500 \\
\hline
\end{tabular}

In posture1, the robot has the maximal step length. In posture6, the torque value of the robot is smallest. Posture2, 3, 4, 5 are randomly selected from joints angle range. And posture7 is the low energy consumption posture according to the weighted sum algorithm. As can be seen from the table, the posture obtained in this paper is the minimum energy consumption under the premise of ensuring the flexibility of the robot. The prototype of low energy static posture is shown in Figure 8.

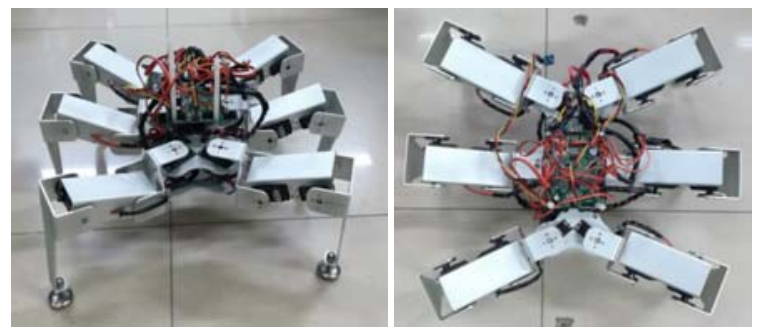

FIGURE VIII. THE PROTOTYPE OF LOW ENERGY STATIC POSTURE

\section{CONCLUSION}

In this paper, the kinematics and statics model of a lowspeed hexapod walking robot is established. According to the characteristics of static standing posture, the low energy standing posture of hexapod robot is presented. Based on the weighted fusion algorithm, a multi-objective optimization function is established to optimize the energy consumption and step length and the low power standing posture is optimized. Finally, the simulation and experimental results show that the proposed posture can meet the requirements of energy consumption and space flexibility which provides theoretical guidance and technical support for the selection of robot prototype driver and robot motion planning.

\section{REFERENCES}

[1] Kottege, Navinda, "Energetics-informed Hexapod Gait Transitions Across Terrains," IEEE International Conference on Robotics \& Automation, 2015, pp.5140-5147.

[2] Lei Zhang, Dedong Li, Fang Yang, "Development and Posture Control of A Hexapod Bionic-Robot,” IEEE International Conference on Robotics and Biomimetics, 2016, pp.77-82.

[3] Yue Zhai, Peng Gao, Yu Sun, "Gait Planning for Multi-motion Mode Wheel-legged Hexapod Robot," IEEE International Conference on Robotics and Biomimetics, 2016, pp.449-454.

[4] D. SanZ-Merodio, E. Garcia, P. Gonzalez-de-Santos, “Analyzing Energy-Efficient Configurations in Hexapod Robots for Demining Applications,” Industrial Robot: An International Journal, 2012, vol.39, pp.357-364.

[5] Bing Bing Yan, Yang Li, Wen Bo Ren, "Modeling and Simulation of Gait Analysis of Hexapod Robot," Applied Mechanics and Materials, 2014, vol.457-458, pp.639-642.

[6] Bo Jin, Cheng Chen, Wei Li, "Power Consumption Optimization for A Hexapod Walking Robot,” Journal of Intelligent \& Robotic Systems, 2013, vol.71, pp.195-209.

[7] HAN J, ZHAO S, LI J, "Movement Gait Harmony Control for Hexapod Robot,” Mechanical \& Electrical Engineering Magazine, 2004, vol.21, pp.8-10.

[8] Erden M S, Kemal L, "Free Generation with Reinforcement Learning for a Six-legged Robot,” Robotics and Autonomous Systems, 2008, vol.56, pp.199-212.

[9] SHIBENDU S R, AJAY K S, DILIP K P, "Analysis of six-legged walking robots," [C]// 14th National Conference on Machines and Mechanisms (NaCoMM09). Durgapur, India:[s.n.], 2009, pp.259-265. 